\title{
Does 29Mhz Micro-Ultrasound Provide Uniform Diagnostic Accuracy Within and Beyond the Peripheral Zone?
}

\author{
Ferdinand Luger*, Andreas Gusenleitner, Jasmin Kaar, Clemens Mayr and Wolfgang Loidl \\ Department of Urology, Ordensklinikum Krankenhaus der Elisabethinen, Austria
}

*Corresponding author: Ferdinand Luger, Department of Urology, Ordensklinikum Krankenhaus der Elisabethinen, Austria.

Received Date: September 11, 2019

Published Date: September 23, 2019

\begin{abstract}
Background: The PRI-MUS ${ }^{\mathrm{TM}}$ (Prostate Risk Identification for Micro-Ultrasound) protocol was developed in 2016 to identify suspicious areas seen by the ExactVu ${ }^{\mathrm{TM}}$ micro-ultrasound imaging platform. While a retrospective validation was performed, no prospective validation has been published in the peer-reviewed literature. Further, this protocol was developed for the peripheral zone and it is unclear whether the accuracy to predict clinically significant cancer is uniform throughout the gland.
\end{abstract}

Methods: 399 prostate biopsies were performed in 372 patients using the ExactVu micro-ultrasound system (Exact Imaging, Markham, Canada) from January 2018 to May 2019 at the Ordensklinikum Linz (Linz, Austria). Subjects had a median PSA of 6.7 (IQR 4.5-11.2) ng/mL and 30\% had positive DRE. Suspicious areas were assessed in real-time using PRI-MUS and a TRUS biopsy was performed in the same session under microultrasound guidance. Biopsies were carried out by 5 providers and results from pathology were then compared with the image findings.

Results: Biopsy pathology confirmed a cancer diagnosis in $60 \%$ of patients, with $42 \%$ of patients harboring Grade Group (GG) $>1$ cancer. The PRI-MUS protocol had an area under the receiver-operator characteristic (AUC) of 0.76 for predicting GG>1 cancer in the peripheral zone. This accuracy varied between 0.68-0.83 depending on prostate region, with highest accuracy in the prostate apex and lowest accuracy in the base. Anterior targets were sampled but generally not assigned a PRI-MUS score as the system is currently only validated in the peripheral zone, still, in the 33/737 anterior samples assigned a PRI-MUS score AUC was 0.80 .

Conclusion: Micro-ultrasound and the PRI-MUS protocol are useful tools to detect cancer and appear to maintain strong diagnostic value throughout the prostate. This technology holds promise for reducing the high false-negative rate of prostate biopsy, without relying on multimodality, multi-specialty solutions like mpMRI.

Keywords: Exact Imaging; mpMRI; ultrasound; Micro-ultrasound; Biopsy; Prostate cancer

Abbreviations: mpMRI: Multiparametric Magnetic Resonance Imaging; PSA: Prostate Specific antigen; PIRADS v2: Prostate Imaging and Reporting Data System Version 2; TRUS: Trans-Rectal Ultrasound; PRI-MUS: Prostate Risk Identification for Micro-Ultrasound

\section{Introduction}

Micro-Ultrasound is a new imaging modality designed as a replacement for conventional transrectal ultrasound (TRUS). Like conventional TRUS, micro-ultrasound uses an endorectal transducer to produce real-time images of the prostate and surrounding tissue, however operating at $29 \mathrm{MHz}$ it does so with a resolution that is 3 -fold higher than conventional systems operating at $6-12 \mathrm{MHz}$. This improved resolution gives micro-ultrasound the ability to image structures down to 70 microns, or the average size of the prostate acini, and so resolve tissue-structure detail which is useful in predicting the presence of cancer.
First presented in 2013 through a study of radical prostatectomy correlation by Pavlovich $\mathrm{CP}$, et al. [1], micro-ultrasound demonstrated promising improvements over conventional ultrasound in both sensitivity and specificity to predict prostate cancer. This work suffered from a lack of structured interpretation, as it was discovered that the appearance of cancer on microultrasound imaging was more diverse than the simple hypoechoic lesion of conventional ultrasound. Ghai S, et al. [2] provided the required protocol in 2016, along with a retrospective validation using data collected from a biopsy cohort [2]. Since that time, other 
work has confirmed the utility of micro-ultrasound in various use cases including screening, replacing conventional ultrasound, supplementing multiparametric Magnetic Resonance Imaging (mpMRI), and focal therapy [3-7].

This work adds to the state of the art in this area by prospectively validating the PRI-MUS protocol on a large cohort of men undergoing biopsy at a single European centre. Data on overall accuracy is provided along with a detailed investigation of targeting accuracy in each segment of the prostate.

\section{Materials and Methods}

399 prostate biopsies were performed in 372 patients using the ExactVu ${ }^{\mathrm{TM}}$ micro-ultrasound system (Exact Imaging, Markham, Canada) from January 2018 to May 2019 at the Ordensklinikum Linz (Linz, Austria). All patients provided informed consent to be included in a prospective database as per institutional policies. Biopsies were carried out by 5 providers using a 14-sample extended sextant protocol with 2-samples directed anteriorly in all patients to rule out large anterior lesions. Additional samples were taken when smaller targets were seen outside of standard positions. A standardized worksheet was used to record PRI-MUS ${ }^{\text {TM }}$ risk level for each area immediately before any biopsy needles had been deployed, in order to minimize the effects of tissue damage on interpretation. Results from pathology were then compared with the image findings.

Subjects had a median age of 66 (IQR 59-73) years, median PSA of 6.7 (IQR 4.5-11.2) ng/mL and 30\% had a suspicious digital rectal exam. MRI data was available in 35 cases beginning November 2018 coincident with the FusionVu micro-ultrasound/MRI fusion feature availability on the ExactVu platform.

\section{Results and Discussion}

Biopsy pathology confirmed a cancer diagnosis in $60 \%$ of patients, with $42 \%$ of patients harboring Grade Group (GG) $>1$ cancer. The PRI-MUS protocol had an area under the receiveroperator characteristic (AUC) of 0.76 for predicting GG>1 cancer. Example PRI-MUS targets are shown in Figure 1. This accuracy varied between 0.68-0.83 depending on prostate region, with highest accuracy in the prostate apex and lowest accuracy in the base. Anterior targets were sampled but generally not assigned a PRI-MUS score as the system is currently only validated in the peripheral zone, still, in the 33/737 anterior samples assigned a PRI-MUS score AUC was 0.80. Full results are shown in Table 1, with graphical representation in sagittal and axial views in (Figure 2\&3).

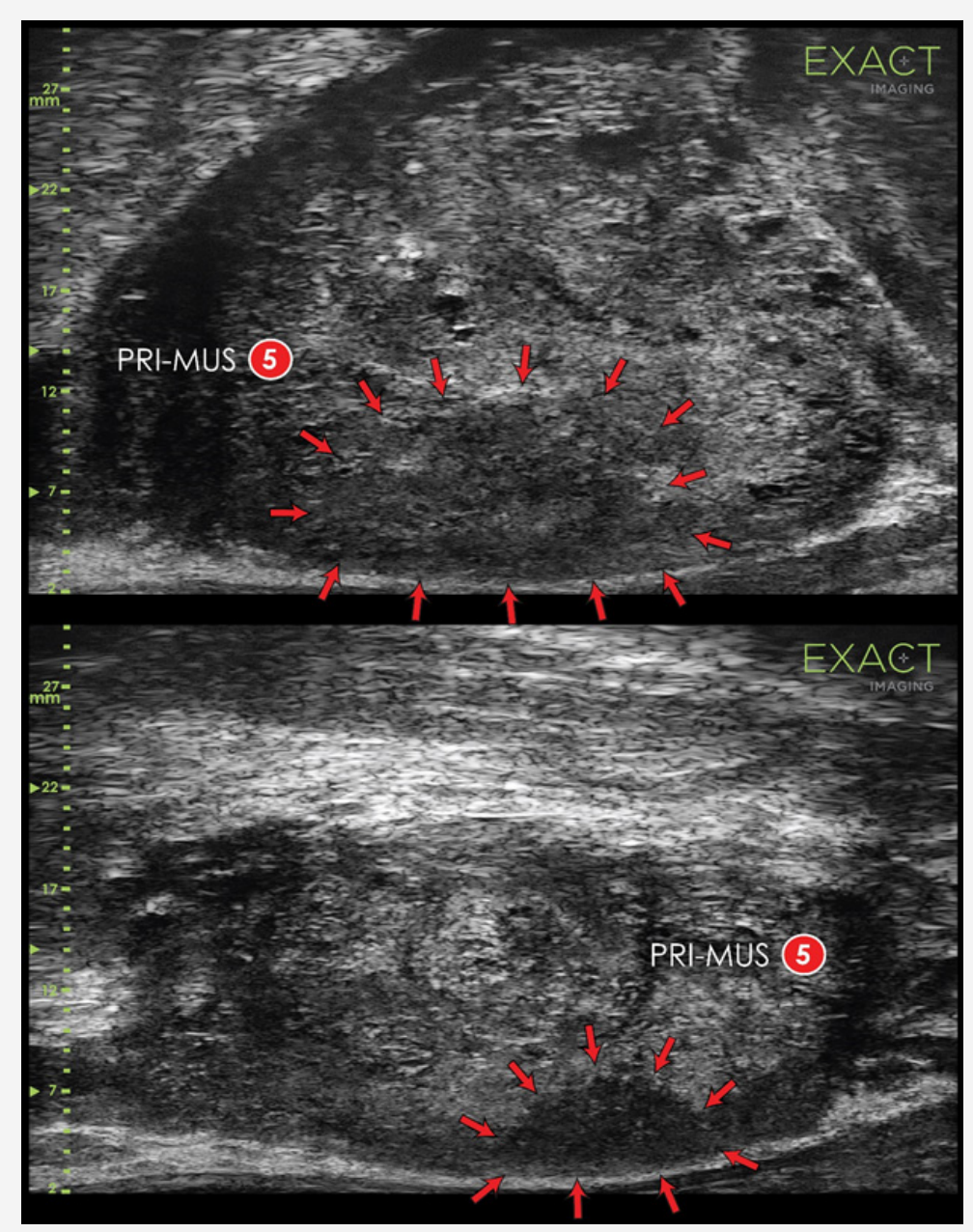

Figure 1: Micro-ultrasound images with PRI-MUS targets in two study patients. (Upper) Smudgy/Mottled PRI-MUS 5 lesion with poorly defined border in otherwise ductal peripheral zone tissue. Biopsy here revealed a Grade Group 3 (Gleason 4+3=7) cancer. (Lower) Mixed echo PRIMUS 5 lesion with well defined border. Biopsy here revealed a Grade Group 5 (Gleason 9) cancer. 


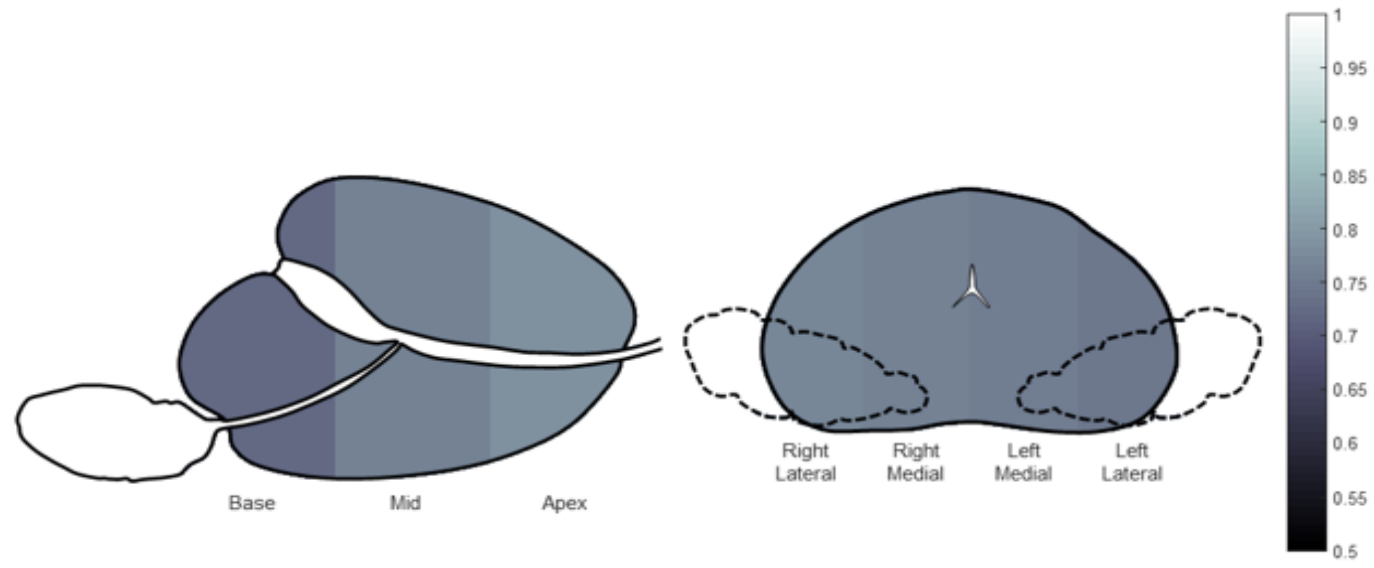

Figure 2: Localization summary. Accuracy (AUC) was relatively uniform throughout, though there was some improvement from base to apex, likely due to anatomical heterogeneity in the base with the presence of central zone/ejaculatory duct and bladder neck muscular tissue. Small variability was also noted from Right lateral to Left lateral, possibly due to ergonomics of transrectal ultrasound scanning.
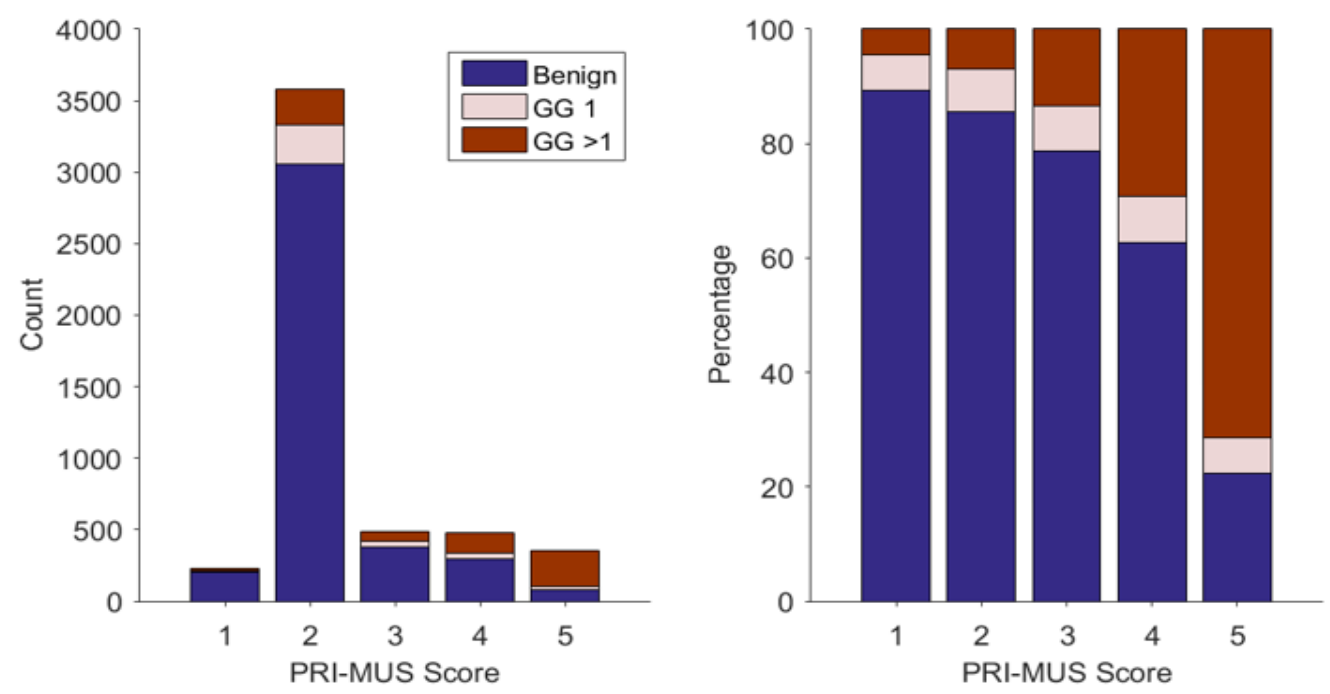

Figure 3: Cancer detection by PRI-MUS Score. The majority of the 5833 samples taken from this cohort were PRI-MUS 2 "hyperechoic with or without ductal patches" tissue. Percentage of cores with each diagnosis is shown in the right side of the figure and demonstrates a clear improvement in detection rate with PRI-MUS score as has been previously reported. Interestingly, the fraction of insignificant cancer is uniform across score.

Table 1: PRI-MUS accuracy by prostate region.

\begin{tabular}{|c|c|c|c|c|c|}
\hline Region & $\begin{array}{c}\text { Number of } \\
\text { Samples }\end{array}$ & $\begin{array}{c}\text { Samples } \\
\text { Scored }\end{array}$ & PCa & csPCa & AUC \\
\hline LAL & 412 & 412 & $22.60 \%$ & $13.80 \%$ & 0.71 \\
\hline LAM & 442 & 442 & $21.70 \%$ & $14.30 \%$ & 0.8 \\
\hline LBL & 407 & 407 & $17.40 \%$ & $11.30 \%$ & 0.75 \\
\hline LBM & 411 & 410 & $17.30 \%$ & $10.70 \%$ & 0.7 \\
\hline LML & 423 & 423 & $23.20 \%$ & $17.00 \%$ & 0.76 \\
\hline LMM & 423 & 423 & $19.60 \%$ & $14.20 \%$ & 0.74 \\
\hline RAL & 440 & 440 & $25.70 \%$ & $15.50 \%$ & 0.79 \\
\hline RAM & 437 & 436 & $22.90 \%$ & $15.10 \%$ & 0.83 \\
\hline RBL & 417 & 417 & $20.90 \%$ & $13.70 \%$ & 0.75 \\
\hline RBM & 421 & 420 & $16.60 \%$ & $12.10 \%$ & 0.68 \\
\hline RML & 424 & 424 & $23.60 \%$ & $13.70 \%$ & 0.77 \\
\hline RMM & 425 & 425 & $24.50 \%$ & $15.10 \%$ & 0.75 \\
\hline Anterior & 737 & 33 & $17.40 \%$ & $9.40 \%$ & 0.8 \\
\hline
\end{tabular}

Overall accuracy here is higher than presented in the Ghai S, et al. [2] retrospective work suggesting a benefit to real-time interrogation of the prostate. Interestingly, the prostatic base has both the lowest PRI-MUS accuracy as well as the lowest rate of clinically significant cancer, suggesting a possible oversensitivity in this region. One hypothesis to be investigated further in future work is the impact of the posterior central zone as it wraps around the ejaculatory duct and bladder neck musculature at the anterior medial base, these structures potentially cause anatomical heterogeneity in the image unrelated to prostate cancer. Similarly, the homogeneity and clarity of anatomy at the apex, particularly in the apical horn may be responsible for the improved accuracy in this region. As described in the original Ghai S, et al. [2] work, a clear increase was noted in risk of significant cancer with PRI-MUS score. This increase did not appear to include insignificant cancer which was largely independent of score. Positive Predictive Value for csPCa ranged from $4.4 \%$ (PRI-MUS 1) to $71.4 \%$ (PRI-MUS 5). 
The unexpectedly high accuracy in the anterior zone is promising for expansion of the protocol to areas outside of the peripheral zone. While it is tempting to draw definite conclusions from this sample it must be emphasized that only a small fraction of anterior zone biopsy cores received scores and bias may have been introduced. Further work on the second version of the PRIMUS protocol will include clear recommendations on sonographic representation of cancer in the anterior portion of the prostate, including rigorous validation.

This study is limited by both population and design. In the first place, the population for biopsy was selected using clinical factors rather than imaging. It is likely that these results would be affected by a change in population either positively, for example if imaging was used to select patients for biopsy, or negatively given a population with a higher burden of benign prostatic diseases or treatment. Design of the study was intended to allow for a large sample rather than a gold-standard reference test. Systematic biopsy is known to miss a significant percentage of prostate cancers. Use of a more invasive test such as template mapping biopsy such as has been performed in other studies Ahmed HU, et al. [8] should be expected to provide more accurate results. This limitation may be alleviated as more patients with MRI and MRI-targeted biopsy are included since MRI-targeted has proven more accurate. Unfortunately, MRItargeted biopsy is still known to miss $15 \%$ of clinically significant prostate cancers Elkhoury FF, et al [9] and it is not clear whether targeted sampling based on one imaging modality would bias results towards or away from micro-ultrasound.

\section{Conclusion}

We presented a prospective analysis of PRI-MUS risk scores over 5833 biopsy samples in 399 micro-ultrasound guided biopsies. Micro-ultrasound and PRI-MUS were accurate across prostate regions, showing some variability from base to apex and unexpectedly high accuracy when used in a small fraction of anterior zone samples. These results suggest that micro-ultrasound may be able to assess risk in the full prostate, subject to constraints on prostate size within the $50 \mathrm{~mm}$ capacity of the system.

Replacing conventional ultrasound with micro-ultrasound offers advantages in detection of clinically significant prostate cancer without significantly affecting the cost or speed of the biopsy procedure. This technology may further improve detection rates when combined with mpMRI as well due to the ability to locate targets in real-time without relying on software fusion, i.e. through real-time visualization. This work confirms the accuracy of micro-ultrasound and PRI-MUS, providing the first evidence of uniformity of accuracy throughout the gland.

\section{Acknowledgement}

None.

\section{Conflicts of Interest}

No conflict of interest.

\section{References}

1. Pavlovich CP, Cornish TC, Mullins JK, Fradin J, Mettee LZ, et al. (2014) High-resolution transrectal ultrasound: Pilot study of a novel technique for imaging clinically localized prostate cancer. Urol Oncol 32(1): 34.e27-e32.

2. Ghai S, Eure G, Fradet V, Hyndman ME, Mc Grath T, et al. (2016) Assessing Cancer Risk on Novel $29 \mathrm{MHz}$ Micro-Ultrasound Images of the Prostate: Creation of the Micro-Ultrasound Protocol for Prostate Risk Identification. J Urol 196(2): 562-569.

3. Lughezzani G, Saita A, Lazzeri M, Paciotti M, Maffei D, et al. (2019) Comparison of the Diagnostic Accuracy of Micro-ultrasound and Magnetic Resonance Imaging/Ultrasound Fusion Targeted Biopsies for the Diagnosis of Clinically Significant Prostate Cancer. Eur Urol Oncol 2(3): $1-4$.

4. Abouassaly R, Klein EA, El Shefai A, Stephenson A (2019) Impact of using $29 \mathrm{MHz}$ high-resolution micro-ultrasound in real-time targeting of transrectal prostate biopsies: initial experience. World J Urol :1-6.

5. Stanton W, Crawford ED, Arangua P, Gretchen Hoyer, Priya N Werahera (2019) Evaluation of the $29 \mathrm{MHz}$ Micro-Ultrasound Imaging for Prostate Cancer Diagnosis and Treatment. Annal Urol \& Nephrol: 1-3.

6. Eure G, Fanney D, Lin J, Wodlinger B, Ghai S (2019) Comparison of conventional transrectal ultrasound, magnetic resonance imaging, and micro-ultrasound for visualizing prostate cancer in an active surveillance population: A feasibility study. Can Urol Assoc J 13(3): E70-E77.

7. Ghai S, Van der Kwast T (2017) Suspicious findings on micro-ultrasound imaging and early detection of prostate cancer. Urol Case Rep 16: 98100.

8. Ahmed HU, El Shater Bosaily A, Brown LC, Gabe R, Kaplan R (2017) Diagnostic accuracy of multi-parametric MRI and TRUS biopsy in prostate cancer (PROMIS): a paired validating confirmatory study. Lancet 389(10071): 815-822.

9. Elkhoury FF, Felker ER, Kwan L, Sisk AE, Delfin M (2019) Comparison of Targeted vs Systematic Prostate Biopsy in Men Who Are Biopsy Naive. JAMA Surg. 